\title{
Trapping a Pentagonal Molecule in a Self-Assembled Molecular Network: an Alkoxylated Isosceles Triangular Molecule Does the Job
}

Received 00th January 20xx,

\author{
Masaru Anzai, ${ }^{a}$ Masahiko Iyoda, ${ }^{\text {b }}$ Steven De Feyter, ${ }^{c}$ Yoshito Tobe ${ }^{\text {d,e }}$ and Kazukuni Tahara*a
}

Accepted 00th January 20xx

DOI: $10.1039 / \times 0 \times x 00000 x$

We herein report a unique example of on-surface adaptive selfassembly. A pentagon-shaped macrocycle, cyclic [5]metaphenyleneacetylene [5]CMPA, is trapped by the adaptive supramolecular network formed by an isosceles triangule, alkoxy substituted dehydrobenzo[14]annulene [14]ISODBA at the liquid/graphite interface, leading to a highly ordered and large-area bicomponent self-assembled molecular network (SAMN), as revealed by scanning tunneling microscopy (STM).

Self-assembled molecular networks (SAMNs) are thin, typically one-molecule-thick films, formed by the self-assembly of molecules on a surface. ${ }^{1}$ Structural control of SAMNs generates a lot of interest in connection with potential applications such as molecular-scale electronics, sensing, and catalysis. ${ }^{2-4}$ SAMN formation has been studied by employing various organic molecular building blocks designed based on supramolecular chemistry principles, to realize sophisticated structural control. ${ }^{5}$ In addition, parameters such as temperature, $, 6,7$ substrate coverage, solute concentration, ${ }^{8,9}$ and electric field ${ }^{10}$ also influence SAMN formation. On atomically flat and conductive substrates, scanning tunneling microscopy (STM) is the method of choice to characterize these SAMNs, also at the liquid/solid interface.

One of the goals is to construct multicomponent networks. Crystalline lattices with up to four different molecules have been reported. ${ }^{11,12}$ These are exceptional cases and bicomponent networks are the most abundant ones. In a substantial number of cases, one of the components acts as a host. ${ }^{13-15}$ This can be at the molecular level, i.e. when a guest is accommodated in the pore of a macrocycle, ${ }^{16-18}$ or at the

a. Department of Applied Chemistry, School of Science and Technology, Meiji University, 1-1-1 Higashimita, Tama-ku, Kawasaki, Kanagawa, 214-8571, Japan b. Graduate School of Science, Tokyo Metropolitan University, 1-1 Minami-osawa, Hachioji, Tokyo 192-0397, Japan.

c. Division of Molecular Imaging and Photonics, Department of Chemistry, KU Leuven, Celestijnenlaan 200 F, 3001 Leuven, Belgium.

d. The Institute of Scientific and Industrial Research, Osaka University, Ibaraki, Osaka 567-0047, Japan.

e. Department of Applied Chemistry, National Chiao Tung University, 1001 Ta Hsueh Road, Hsinchu 30030, Taiwan.

Electronic Supplementary Information (ESI) available: Experimental details, additional STM images, details of MM simulations, energy estimations, characterization data of new compounds. See DOI: 10.1039/x0xx00000x supramolecular level,19-21 i.e. when the arrangement of host molecules creates a supramolecular pore. Shapecomplementary, i.e. between the cavity provided by the molecular or supramolecular host network and the guest, is an important element as it may maximize the van der Waals interactions between the guest and the rim of the pore, thereby stabilizing the host-guest networks. Furthermore, a structural change of the host pore via an induced-fit mechanism, ${ }^{22-26}$ may aid in trapping guest molecules, even if by design and prior to this adaptation, there is no perfect fit between guest and host. Such effect is most often observed for supramolecular hosts. Despite of the successes in trapping for instance individual diskshaped, triangular, or square molecules, or molecular complexes with six-fold symmetry, trapping five-fold diskshaped molecules in a crystalline network based on host-guest strategies hasn't been successful yet. In general, there is a need of formulating clear design principles for the co-crystallization of pentagonal molecules, based on the host-guest principle.

In this work, we develop such a strategy, and implement it for the particularly difficult case of pentagonal molecules. Pentagon-shaped objects have roused immense interest in areas ranging from art, mathematics, material physics to molecular science. ${ }^{27-30}$ Trapping of pentagonal molecules in a host network is notoriously challenging. Only one study reports on the co-assembly of a functionalized non-planar pentagonal molecule using host-guest principles, selecting a pentagonal guest in view of the supramolecular phases formed by the host. ${ }^{31}$ This can be explained by the fact that from a design point of view it is quite challenging to come up with a system, molecular as well as supramolecular, that fulfills the geometric requirements for trapping of pentagonal planar molecules, in particular if they are large.

While the molecular approach may be attractive in some cases, in general it is rather limited in scope as extending or tuning the size of polygonal macrocycles is not trivial. We believe that SAMNs with the potential to host guest molecules are a better and more versatile alternative. In this Communication, we report on our efforts to capture a diskshaped pentagonal molecule using SAMNs, following the hostguest strategy, with the ambition to form an extended crystalline bi-component supramolecular network. 
(a)

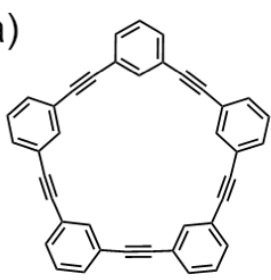

(b)

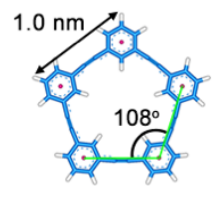

[5]CMPA

(c)

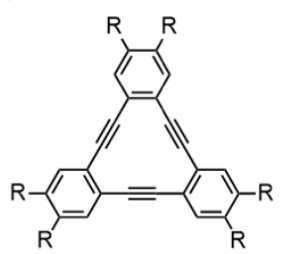

[12]DBA, $\mathrm{R}=\mathrm{OC}_{10} \mathrm{H}_{21}$

(e)

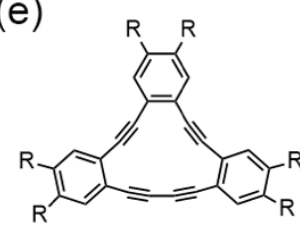

[14]ISODBA, $\mathrm{R}=\mathrm{OC}_{10} \mathrm{H}_{21}$ (d)

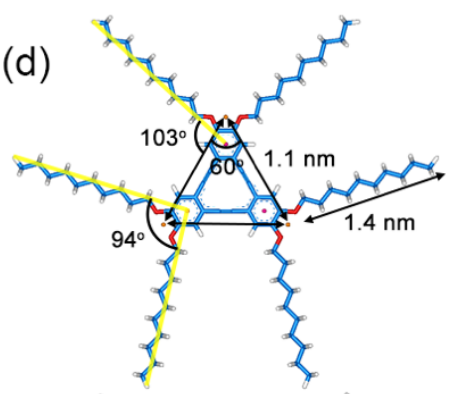

(f)

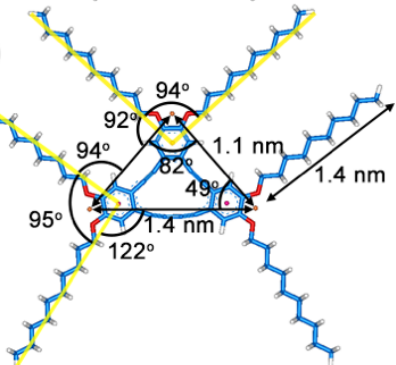

Figure 1. (a, c, e) Chemical structures of [5]CMPA, [12]DBA and [14]ISODBA. (b, d, f) Optimized molecular models of [5]CMPA (b), [12]DBA (d) and [14]ISODBA (f) by DFT calculations (B3LYP/6-31g(d)) with $D_{5 h}, D_{3 h}$ and $C_{2 v}$ symmetric constraints. Green lines in (b) connect the centroids (pink dots) of the benzene rings. Orange dots in (d, f) are the median points between the two oxygen atoms attached to the same benzene ring. Yellow lines in $(d, f)$ connect the terminal hydrogen atoms of the alkoxy chains and the centroids of the benzene rings that represent alkoxy chain orientations.

As a pentagonal shaped molecule, we chose [5]CMPA because of its planar and rigid pentagonal $\pi$-core. ${ }^{32}$ Furthermore, it is also large enough to be clearly identified by STM. Structural optimization of [5]CMPA by a DFT calculation (B3LYP/6-31g(d)) in vacuum confirms that it adopts a regular $D_{5 h}$ symmetric structure with side lengths of $1.0 \mathrm{~nm}$ and vertex angles of $108^{\circ}$ (Fig. 1b). We took these structural characteristics into consideration upon designing or selecting a potential host.

To trap this pentagonal molecule, we considered the wellknown class of alkoxylated equilateral dehydrobenzo[12]annulene [12]DBA, and a new class of alkoxylated isosceles [14]ISODBA, both with decyloxy groups, based on DFT optimized structures in vacuum (Fig. 1d,f). The length of the decyloxy group $(1.4 \mathrm{~nm})$ is somewhat longer than the side of [5]CMPA to ensure good van der Waals contacts. The lengths of equilateral sides of [12]DBA and isosceles sides of [14]ISODBA are $1.1 \mathrm{~nm},{ }^{33}$ and are similar to the length of the side of [5]CMPA $(1.0 \mathrm{~nm})$. The longest side of [14]ISODBA measures $1.4 \mathrm{~nm}$, which will be important to interact with [5]CMPA by taking the spacing of the alkoxy substituents into account. [12]DBA and [14]ISODBA differ in the internal angles of the $\pi$-cores ( $60^{\circ}$ for [12]DBA, $82^{\circ}$ and $49^{\circ}$ for [14] ISODBA), and the angles between the alkoxy chains and the sides of the triangles $\left(103^{\circ}\right.$ for [12]DBA, and $92^{\circ}, 94^{\circ}$ and $122^{\circ}$ for

[14]ISODBA). The angles between the alkoxy chains attached to the same benzene ring are similar for [12]DBA $\left(94^{\circ}\right)$ and [14]ISODBA $\left(94^{\circ}\right.$ and $\left.95^{\circ}\right)$. As previously reported, [12]DBA forms a honeycomb structure on graphite, in which the angles between the alkoxy chains are ca. $120^{\circ}$ and the chains follow the main symmetry axes of the graphite substrate (Fig. S1g, ESI). ${ }^{19,20}$ On the other hand, the $C_{2 v}$ symmetric core of [14]ISODBA may cause epitaxial mismatch with respect to the graphite surface, thereby increasing the flexibility in the alkoxy chain orientation. Moreover, the large angle of $122^{\circ}$ between the alkoxy chains and the long side of [14]ISODBA is potentially a beneficial structural element for accommodating [5]CMPA. These features of [14]ISODBA may be crucial, if we consider [5]CMPA to be sandwiched by the alkoxy chains (Fig. S1h,i, ESI).

Synthesis of [5]CMPA was reported before. ${ }^{32}$ [14]ISODBA was newly synthesized according to the procedure reported before (See ESI). ${ }^{33,34}$

[5]CMPA and [14]ISODBA were dissolved in 1,2,4trichlorobenzene (TCB). The concentration of [5]CMPA is $2.0 \times$ $10^{-3} \mathrm{M}$. Several solutions were prepared for [14]ISODBA $(2.0 \times$ $10^{-4}, 2.0 \times 10^{-5}$ and $2.0 \times 10^{-6} \mathrm{M}$ ) to investigate the effect of solute concentration on SAMN formation..$^{8,9}$ Also mixtures of [14]ISODBA $\left(1.1 \times 10^{-5} \mathrm{M}\right)$ and [5]CMPA $\left(4.4 \times 10^{-4} \mathrm{M}\right)$ as well as [12]DBA $\left(1.1 \times 10^{-5} \mathrm{M}\right)$ and [5]CMPA $\left(4.4 \times 10^{-4} \mathrm{M}\right)$ were prepared. TCB was selected as solvent because of the good solubility of the components. Highly oriented pyrolytic graphite (HOPG) was employed as a conductive substrate. The SAMNs were prepared by dropping the solution of the compound(s) onto the substrate (10 or $15 \mu \mathrm{L}$ ). For annealing treatment, the solution of the compound(s) was/were poured to a liquid cell placed on the substrate $(20$ or $40 \mu \mathrm{L})$. The annealing treatment was applied at $80^{\circ} \mathrm{C}$ for $3 \mathrm{~h}$ in a sealed sample drying oven. All STM observations were conducted at the TCB/graphite interface at room temperature.

First, we attempted STM observations of SAMNs formed by [5]CMPA. However, no monolayer formation of [5]CMPA was observed, even at the high concentration. ${ }^{30}$ On the other hand, [14]ISODBA forms three structures, Phases 1-3 (Fig. 2, Tables S1 and S2, ESI) at different concentrations, in contrast to [12]DBA which only forms a honeycomb type structure in the same concentration range. ${ }^{8,19}$ Details on the impact of concentration and annealing treatment can be found in ESI. In STM imaging, $\pi$-conjugated cores are often resolved as bright features, corresponding to an increased tip-sample distance in quasiconstant current mode, because of their higher tunneling efficiency. ${ }^{35}$ Therefore, we can safely assign the bright triangular features in the STM images to the $\pi$-conjugated core of [14]ISODBA. Dim lines connecting the triangular $\pi$-cores are adsorbed alkyl chains. In Phases 1-3, [14]ISODBA forms molecular zigzag rows as a basic structural motif, with interdigitated alkyl chains $([1+1]$ or [2+2] type interactions, see ESI). ${ }^{19,36}$ The spacing between the molecular rows differs depending on the surface molecular density. The dim spots between the molecular zigzag rows are attributed to coadsorbed TCB molecules (Fig. S3, ESI). Lowering symmetry of the $\pi$-core to an isosceles triangle has a clear impact on SAMN formation, as [14]ISODBA, in contrast to [12]DBA, doesn't form 
a porous honeycomb structure. An intriguing aspect of [14]ISODBA is the adaptability of its alkoxy chains, as indicated by the phase dependent relative orientation of the adsorbed alkyl chains with respect to the main symmetric axes of graphite (angles $\alpha$ and $\beta$ in Fig. 2). This is again in contrast with the honeycomb structure of [12]DBA, where all adsorbed interdigitated alkyl chains are parallel to the main symmetry axes of the graphite.
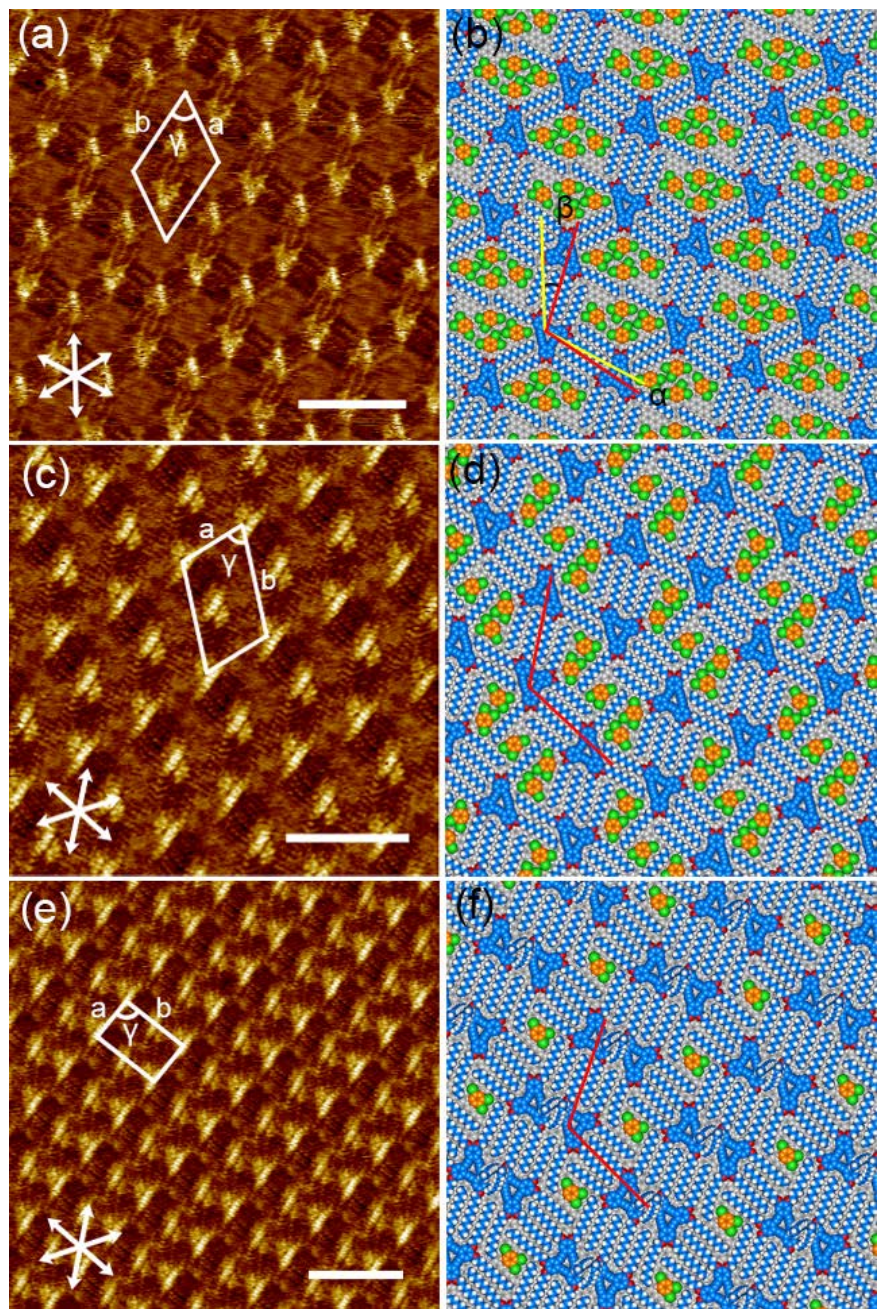

Figure 2. (a) STM image of Phase 1 formed by [14]ISODBA at the 1,2,4-trichlorobenzene (TCB)/graphite interface (concentration; $2.0 \times 10^{-5} \mathrm{M}$, tunneling parameters; $I_{\text {set }}=200$ $\mathrm{pA}, V_{\text {bias }}=-0.31 \mathrm{~V}$, unit cell parameters; $\mathrm{a}=4.0 \pm 0.1 \mathrm{~nm}, \mathrm{~b}=4.7 \pm 0.1 \mathrm{~nm}, \gamma=55 \pm 1^{\circ}$ the angles between the alkyl chain orientations and main symmetric axes of graphite; $\alpha$ $=5 \pm 2^{\circ}$ and $\beta=26 \pm 3^{\circ}$ ). (b) Molecular model of Phase 1 optimized by molecular mechanics (MM) simulation (periodic boundary conditions (PBCs); $a=4.1 \mathrm{~nm}, \mathrm{~b}=4.7$ $\mathrm{nm}, \gamma=54^{\circ}$ ). (c) STM image of Phase 2 at the TCB/graphite interface (concentration; 2.0 $\times 10^{-4} \mathrm{M}$, tunneling parameters; $I_{\text {set }}=300 \mathrm{pA}, V_{\text {bias }}=-0.39 \mathrm{~V}$, unit cell parameters; $a=2.9$ $\pm 0.1 \mathrm{~nm}, \mathrm{~b}=4.8 \pm 0.2 \mathrm{~nm}, \gamma=75 \pm 2^{\circ}$, angles; $\alpha<3^{\circ}$ and $\beta<3^{\circ}$ ). (d) Molecular model of Phase 2 optimized by MM simulation (PBCs; $a=2.9 \mathrm{~nm}, b=4.7 \mathrm{~nm}, \gamma=74^{\circ}$ ). (e) STM image of Phase $\mathbf{3}$ at the TCB/graphite interface at room temperature (concentration; 2.0 $\times 10^{-4} \mathrm{M}$, tunneling parameters; $I_{\text {set }}=300 \mathrm{pA}, V_{\text {bias }}=-0.31 \mathrm{~V}$, unit cell parameters; $\mathrm{a}=2.5$ $\pm 0.1 \mathrm{~nm}, \mathrm{~b}=3.9 \pm 0.1 \mathrm{~nm}, \gamma=89 \pm 2^{\circ}$ ). (f) Molecular model of Phase 3 optimized by MM simulation (PBCs; $a=2.6 \mathrm{~nm}, b=3.8 \mathrm{~nm}, \gamma=88^{\circ}$, angles; $\alpha<3^{\circ}$ and $\beta<3^{\circ}$ ). Scale bars in (a, c, d) correspond to $5 \mathrm{~nm}$. Red lines and yellow lines in (b, d, f) represent the orientations of the adsorbed alkyl chains and the main symmetric axes of graphite respectively. In (b, d, f), carbon atoms of [14]ISODBA are colored in blue. Carbon and chlorine atoms of the co-adsorbed TCB molecules are colored in orange and light green, respectively.
At first sight, these results don't look promising to form host networks to accommodate [5]CMPA. In particular, the lack of pentagonal pores might seem problematic. However, one should not ignore the level of adaptivity these systems may show in response to the adsorption of [5]CMPA, in particular for the [14]ISODBA/[5]CMPA system based on their structural similarities (vide supra).

First, we have attempted the co-assembly formation using pre-mixed solution of [12]DBA and [5]CMPA in TCB. As expected, [12]DBA only forms the honeycomb structure (Fig. S4 and S5, ESI). Yet the pores sometimes appear fuzzy and brighter, the molecular shape of [5]CMPA was only identified at a few distorted hexagonal pores (defects, Fig. S4). Annealing treatment did not alter the outcome. This is attributed to the shape mismatch between the pore and guest molecule.

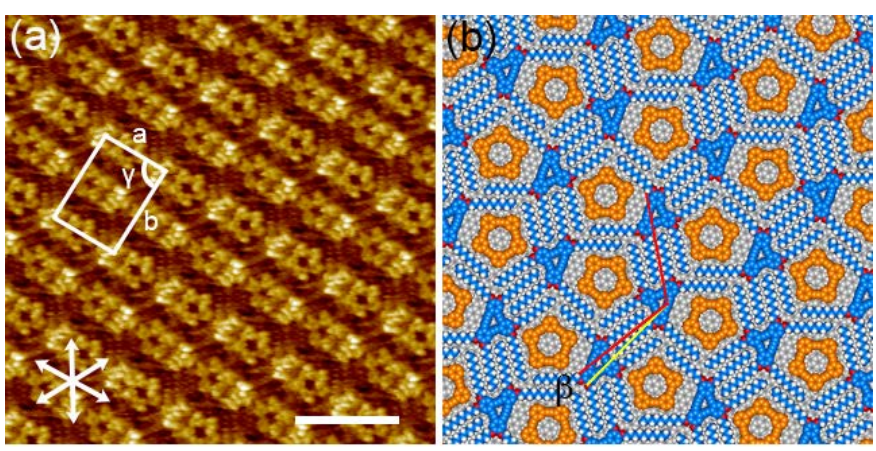

Figure 3. (a) STM image of Co-crystal (concentration; [14]ISODBA: $1.1 \times 10^{-5} \mathrm{M}$ [5]CMPA: $4.4 \times 10^{-4} \mathrm{M}$, tunneling parameters; $I_{\text {set }}=100 \mathrm{pA}, V_{\text {bias }}=-0.62 \mathrm{~V}$, unit cell parameters; $a=3.4 \pm 0.1 \mathrm{~nm}, b=4.7 \pm 0.1 \mathrm{~nm}, \gamma=89 \pm 1^{\circ}$, angles; $\alpha<3^{\circ}$ and $\beta=10 \pm 4^{\circ}$ ). The unit cell area is $16 \pm 1 \mathrm{~nm}^{2}$. Scale bar corresponds to $5 \mathrm{~nm}$. (b) Network model of the Co-crystal which is optimized by a MM simulation (PBCs: $a=3.3 \mathrm{~nm}, \mathrm{~b}=4.7 \mathrm{~nm}, \gamma=89^{\circ}$ ). Red lines and a yellow line in (b) represent orientations of alkyl chains and the main symmetric axis of graphite, respectively. Carbon atoms of [14]ISODBA and [5]CMPA in (b) are colored in blue and orange, respectively.

A completely different outcome was observed for the mixture of [14]ISODBA and [5]CMPA. With or without annealing treatment, the exclusive formation of large domains of a structurally new SAMN was observed, in which both [5]CMPA and [14]ISODBA can be clearly discerned (Fig. 3a and S6, ESI). In this bicomponent Co-crystal, two alkyl chains at both short sides of the triangular core of [14]ISODBA form a [2+2] type alkyl chain interdigitation pattern with adjacent [14]ISODBA molecules producing a zigzag molecular row unlike the hexagonal pattern formed by [12]DBA. The other two alkyl chains connect the zigzag molecular rows via [1+1] interactions, thereby bisecting the channel to form a non-hexagonal space. The bright pentagonal cores of [5]CMPA are clearly resolved in between the zigzag molecular rows, in which the four sides of the pentagon are surrounded by the alkyl chains. The angles between orientations of interdigitated $[2+2]$ type alkyl chains and main symmetry axes of graphite are $\alpha<3^{\circ}$ and $\beta=10 \pm 4^{\circ}$, respectively (Table S2, ESI), showing the adaptability of the alkyl chains, which is crucial for the formation of Co-crystal. Note that also the same Co-crystal is formed in 1-phenyloctane (Fig. S7, ESI), suggesting that the solvent does not play an important role for its preferential formation. 
Since the guest induced structural change was not observed for a mixture of [12]DBA and [5]CMPA, the structural recognition of [5]CMPA at the long side of [14]ISODBA $(1.4 \mathrm{~nm})$ to [5]CMPA $(1.0 \mathrm{~nm})$ and adaptability of the alkoxy chains are considered crucial elements, to maximize van der Waals interactions between the host and guest molecules. Here we briefly discuss the thermodynamic stability of the co-assembly based on the comparison between Phase $\mathbf{1}$ and Co-crystal as both appear in the same low-concentration range in TCB and have the same unit cell area $\left(16 \mathrm{~nm}^{2}\right.$, Table S2, ESI).

Interaction energies of Phases 1-3 and Co-crystal are analyzed by molecular mechanics (MM) simulations (see ESI). ${ }^{36,37}$ As a result, $\Delta h$ values that are enthalpy changes per unit area upon formation of each phase become -23.5 and $21.5 \mathrm{kcal} \cdot \mathrm{mol}^{-1} \cdot \mathrm{nm}^{-2}$ for Phase 1 and Co-crystal. This estimate favoring Phase 1 does not support the experimental observation where Co-crystal was formed preferably to Phase 1. Note that this enthalpy estimation does not include solvation and also wetting energies, that would influence Co-crystal formation. ${ }^{38,39}$ Another plausible factor is the difference in entropy loss upon SAMNs formation. The numbers of the coadsorbed TCB and [5]CMPA molecules per the unit cells are different between Phase 1 and Co-crystal (8 vs. 2).

In conclusion, we herein reported the formation and stable co-assembly of pentagonal shaped molecule [5]CMPA and isosceles triangle, alkoxy substituted [14]ISODBA at the $\mathrm{TCB} /$ graphite interface. In addition to the structural recognition of [5]CMPA at the long side of [14]ISODBA, the adaptability of alkyl chain's orientation due to de-symmetrized core of [14]ISODBA is a key element for the formation of Co-crystal. Present study provides useful information for structural control of self-assembled systems formed by pentagonal molecules, which is of importance in the field of 2D crystal engineering.

This work was supported by JSPS KAKENHI Grant Numbers JP17H04794 and JP17K19130, the Fund of Scientific Research Flanders (FWO), and KU Leuven - Internal Funds.

\section{Conflicts of interest}

There are no conflicts to declare.

\section{Notes and references}

1 D. P. Goronzy, M. Ebrahimi, F. Rosei, Arramel, Y. Fang, S. De Feyter, S. L. Tait, C. Wang, P. H. Beton, A. T. S. Wee, P. S. Weiss and D. F. Perepichka, ACS Nano, 2018, 12, 7445.

2 J. V. Barth, G. Costantini, and K. Kern, Nature, 2005, 437, 671.

3 C.-A. Palma, and P. Samorì, Nat. Chem., 2011, 3, 431.

4 J. V. Barth, Annu. Rev. Phys. Chem., 2007, 58, 375.

5 J. A. A. W. Elemans, S. Lei and S. De Feyter, Angew. Chem. Int. Ed., 2009, 48, 7298

6 M. O. Blunt, J. Adisoejoso, K. Tahara, K. Katayama, M. Van der Auweraer, Y. Tobe and S. De Feyter, J. Am. Chem. Soc., 2013, 135, 12068

7 A. Bellec, C. Arrigoni, G. Schull, L. Douillard, C. FioriniDebuisschert, F. Mathevet, D. Kreher, A.-J. Attias and F. Charra, J. Chem. Phys., 2011, 134, 124702.
8 S. Lei, K. Tahara, F. C. De Schryver, M. Van der Auweraer, Y. Tobe and S. De Feyter, Angew. Chem. Int. Ed., 2008, 47, 2964.

9 A. Ciesielski, P. J. Szabelski, W. Rżysko, A. Cadeddu, T. R. Cook, P. J. Stang and P. Samorì, J. Am. Chem. Soc., 2013, 135, 6942.

10 S.-B. Lei, K. Deng, Y.-L. Yang, Q.-D. Zeng, C. Wang and J.-Z. Jiang, Nano Lett., 2008, 8, 1836.

11 J. Adisoejoso, K. Tahara, S. Okuhata, S. Lei, Y. Tobe and S. De Feyter, Angew. Chem. Int. Ed., 2009, 48, 7353.

12 Y. Xue and M. B. Zimmt, J. Am. Chem. Soc., 2012, 134, 4513.

13 J. A. Theobald, N. S. Oxtoby, M. A. Phillips, N. R. Champness and P. H. Beton, Nature, 2003, 424, 1029.

14 D. Bonifazi, S. Mohnani and A. Llanes-Pallas, Chem. Eur. J., 2009, 15, 7004 .

15 X. Zhang, Q. Zeng and C. Wang, Sci. China Chem., 2014, 57, 13.

16 K. Tahara, S. Lei, W.Mamdouh, Y. Yamaguchi, T. Ichikawa, H. Uji-i, M. Sonoda, K. Hirose, F. C. De Schryver, S. De Feyter and Y. Tobe, Y. J. Am. Chem. Soc., 2008, 130, 6666.

17 B. Schmaltz, A. Rouhanipour, H. J. Räder, W. Pisula and K. Müllen, Angew. Chem. Int. Ed., 2009, 48, 720.

18 M. Shen, Z. Luo, S. Zhang, S. Wang, L. Cao, Y. Geng, K. Deng, D. Zhao, W. Duan and Q. Zeng, Nanoscale, 2016, 8, 11962.

19 K. Tahara, S. Furukawa, H. Uji-i, T. Uchino, T. Ichikawa, J. Zhang, W. Mamdouh, M. Sonoda, F. C. De Schryver, S. De Feyter and Y. Tobe, J. Am. Chem. Soc., 2006, 128, 16613.

20 Y. Tobe, K. Tahara and S. De Feyter, Bull. Chem. Soc. Jpn., 2016, 89, 1277.

21 M. Lackinger and W. M. Heckl, Langmuir, 2009, 25, 11307.

22 S. Furukawa, K. Tahara, F. C. De Schryver, M. Van Der Auweraer, Y. Tobe and S. De Feyter, Angew. Chem. Int. Ed., 2007, 46, 2831

23 D. Bléger, D. Kreher, F. Mathevet, A.-J. Attias, G. Schull, A Huard, L. Douillard, C. Fiorini-Debuischert, F. Charra, Angew. Chem. Int. Ed., 2007, 46, 7404.

24 M. Blunt, X. Lin, M. del C. Gimenez-Lopez, M. Schröder, N. R. Champness and P. H. Beton, Chem. Commun., 2008, 2304.

25 J. Sun and S. Lei, J. Phys. Chem. C, 2018, 122, 12315.

26 M. Li, P. Xie, K. Deng, Y.-L. Yang, S.-B. Lei, Z.-Q. Wei, Q.-D. Zeng, C. A. Wang, Phys. Chem. Chem. Phys., 2014, 16, 8778.

27 N. A. Wasio, R. C. Quardokus, R. P. Forrest, C. S. Lent, S. A. Corcelli, J. A. Christie, K. W. Henderson and S. A. Kandel, Nature, 2014, 507, 86.

28 T. Bauert, L. Merz, D. Bandera, M. Parschau, J. S. Siegel and K. H. Ernst, J. Am. Chem. Soc., 2009, 131, 3460.

29 B. E. Hirsch, S. Lee, B. Qiao, C.-H. Chen, K. P. McDonald, S. L. Tait and A. H. Flood, Chem. Commun., 2014, 50, 9827.

30 K. Tahara, T. Balandina, S. Furukawa, S. De Feyter and Y. Tobe, CrystEngComm, 2011, 13, 5551.

31 B. Calmettes, S. Nagarajan, A. Gourdon, M. Abel, L. Porte and R. Coratger, Angew. Chem. Int. Ed., 2008, 47, 6994.

32 J. Yamakawa, M. Ohkoshi, F. Takahashi, T. Nishiuchi, Y. Kuwatani, T. Nishinaga, M. Yoshida and M. Iyoda, Chem. Lett., 2008, 37, 784.

33 K. P. Baldwin, A. J. Matzger, D. A. Scheiman, C. A. Tessier, K. P. C. Vollhardt and W. J. Youngs, Synlett, 1995, 1215.

34 Q. Zhou, P. J. Carroll and T. M. Swager, J. Org. Chem., 1994, 59, 1294.

35 R. Lazzaroni, A. Calderone, G. Lambin, J. P. Rabe and J. L. Brédas, Synth. Met., 1991, 41, 525.

36 R. Gutzler, T. Sirtl, J. F. Dienstmaier, K. Mahata, W. M. Heckl, M. Schmittel and M. Lackinger, J. Am. Chem. Soc., 2010, 132, 5084.

37 K. Tahara, R. Nakayama, M. Maeda, S. De Feyter and Y. Tobe, J. Phys. Chem. C, 2019, 123, 27020.

38 W. Song, N. Martsinovich, W. M. Heckl and M. Lackinger, J. Am. Chem. Soc., 2013, 135, 14854

39 R. A. Barnard and A. J. Matzger, Langmuir, 2014, 30, 7388. 